\title{
Ein neues, solares Zeitalter hat begonnen
}

\author{
Vor gut zehn Jahren entstand die Idee, mit riesigen Solarkraftwerken erneuerbaren Strom \\ in den Wüsten Nordafrikas und Vorderasiens zu produzieren - sowohl für die lokale als auch \\ für die europäische Stromversorgung. Inzwischen hat die Energiewende in der Wüste begonnen, \\ allerdings anders als ursprünglich geplant: Nicht solarthermische Großkraftwerke, \\ sondern die Fotovoltaik setzt sich mehr und mehr durch.
}

Hartmut Graßl

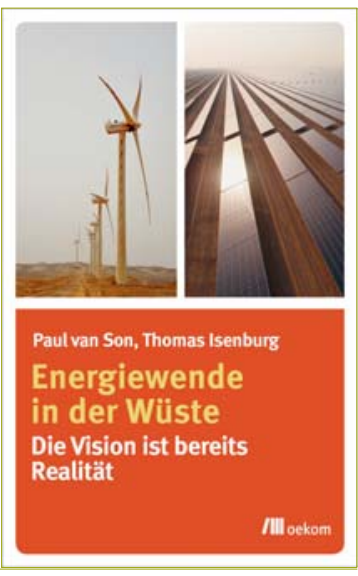

van Son, P., T. Isenburg. 2019. Energiewende in der Wüste. Die Vision ist bereits Realität. München: oekom. 264 S., 26,- EUR, ISBN 978-3-96238030-4

ie Menschheit braucht bei noch immer steigender Bevölkerung und pro Kopf wachsendem Wohlstand immer mehr Energie. In über 99 Prozent seiner Existenz hat der Homo sapiens erneuerbare Energien wie Sonne, Holz, Wasser- und Windkraft genützt; erst seit wenigen Jahrhunderten „plündert“ er die Reste von Sonnenenergie in den sehr alten Ablagerungen in der Erdkruste, indem er Kohle, Erdöl und Erdgas mit hoher Geschwindigkeit im Vergleich zur Neubildung verbrennt. Trotz des stark gestiegenen Energiehungers ist die Energieflussdichte (Energie pro Zeit- und Flächeneinheit) im Vergleich zum Angebot der Sonne glücklicherweise noch sehr gering. Sogar im stark bewölkten Deutschland in höheren mittleren Breiten mit hoher Bevölkerungsdichte werden an der Erdoberfläche im Jahresmittel noch 110 Watt pro Quadratmeter (W/m²) von der Sonne angeboten, allerdings auch nur etwa $1,5 \mathrm{~W} / \mathrm{m}^{2}$ genutzt - und dies immer noch zu etwa vier Fünfteln aus den fossilen Brennstoffen. Von den global von der Sonne im Mittel angebotenen rund 160 $\mathrm{W} / \mathrm{m}^{2}$ an der Erdoberfläche bräuchten wir Menschen zurzeit 0,03 W/m² für unsere Energieinfrastruktur. Auch bei komplettem

Prof. Dr. Hartmut Graßl | Max-Planck-Institut für Meteorologie | Hamburg | Deutschland | hartmut.grassl@mpimet.mpg.de

(c) 2020 H. Graßl; licensee oekom verlag. This article is distributed under the terms of the Creative Commons Attribution License CCBY 4.0 (http://creativecommons.org/licenses/by/4.0). https://doi.org/10.14512/gaia.29.3.9

Submitted September 7, 2020; revised version accepted September 14, 2020 (editorial board peer review).
Ausstieg aus fossilen Brennstoffen müssten wir also nur einen sehr kleinen Teil der Sonnenenergie vor der Absorption am Boden oder dem oberen Ozean sowie in der Vegetation abzweigen.

\section{Desertec und die Idee vom Wüstenstrom}

Es war also folgerichtig, als der Physiker Gerhard Knies in Hamburg 2008 mit der Desertec-Initiative darauf hinwies, dass die Sonnenenergie in Form von elektrischem Strom aus den Wüsten locker Europa und viele andere Länder versorgen könnte. ${ }^{1}$ Auch der Transport der Energie aus den sonnigen und trockenen Regionen über Stromleitungen sowie Wasserstoff und andere Flüssigkeiten in Röhren und Schiffen wurde schon damals angesprochen, genauso wie die Abschätzung, dass das Angebot von der Sonne von $45^{\circ}$ nördliche Breite bis in die Wüstenzone um etwa $20^{\circ}$ nördliche Breite nur etwa um den Faktor 2 ansteigt.

Die bisher geschilderten physikalischen Randbedingungen werden im Buch Energiewende in der Wüste von van Son und Isenburg als gegeben betrachtet. Die Autoren zeigen aber nicht, dass bei sehr niedrigen Kosten für eine Kilowattstunde Strom aus der Fotovoltaik (jetzt bereits erreicht) die Transportinfrastruktur die größten Kosten verursacht. Also könnte dann nur ein kleiner oder gar kein Anreiz für Energietransporte über große Entfernungen und über das existierende europäische Stromnetz hinausgehend vorhanden sein. Auch weil bei höherer Windgeschwindigkeit im Winter der Windstrom bei niedrig stehender
Sonne in den mittleren und hohen Breiten die Einbußen oft mehr als kompensieren kann.

Das Buch dreht sich um die Entwicklung der im Oktober 2009 gegründeten Desertec Industrial Initiative (Dii), einer $\mathrm{GmbH}$, zunächst mit Sitz in München, jetzt in Dubai, die mit sehr großem Medienecho startete und deren Hauptzweck darin bestand, für die europäischen Bürger(innen) den Weg für den Strom aus der Wüste nach Europa zu bereiten - wozu damals Investitionen von extrem hoch erscheinenden 400 Milliarden Euro abgeschätzt wurden. Der Geschäftsführer der Dii und Hauptautor des Buchs, Paul van Son, schildert zusammen mit dem Journalisten Thomas Isenburg teilweise minutiös, wie aus dem ursprünglichen Traum der großen Industriebetriebe in der Dii, vorwiegend aus Europa mit einem zunächst deutschen Schwerpunkt, eine kleine $\mathrm{GmbH}$ wurde, die nach heftigen internen Debatten - jetzt in Dubai ansässig - mithalf, dass die erneuerbaren Energieträger Sonne und Wind in den meisten MENA-Ländern (Middle East and North Africa) Fuß fassen konnten, mit dem Leuchtturm Marokko. Der Untertitel des Buchs Die Vision ist bereits Realität ist also nur eine leichte Übertrei-

1 Die ersten Gedanken und Modellrechnungen dazu machten sich allerdings schon in den 1970er Jahren einige andere (Williams et al. 1977). 
bung. ${ }^{2}$ Doch nirgendwo sind Stromexporte in Nachbarländer wesentlich oder gar von MENA nach Europa im Bau.

\section{Energiewende und Paris Agreement}

Den entscheidenden Anstoß für die Trendwende zu den Erneuerbaren in MENA gab das seit November 2016 völkerrechtlich verbindliche Übereinkommen von Paris (Paris Agreement), das den Ausstieg aus den fossilen Energien weltweit eingeläutet hat; und das ihn bis $2050 \mathrm{im}$ Wesentlichen vollendet sehen will. Dass bei diesem Schwenk zu den erneuerbaren Energieträgern die zunächst favorisierten solar-thermischen Kraftwerke (etwa Parabolrinnen-Kraftwerke) gegenüber der Fotovoltaik in Bezug auf die Kosten verloren haben - wie es auch van Son feststellt, ist leicht erklärbar und hätte vorausgesehen werden können: Die Techniken für thermische Kraftwerke sind schon weitgehend ausgereift, während eine nur um ein Prozent höhere Umwandlungsrate von Sonnenstrahlung in elektrischen Strom in den Solarzellen, bei bisher im Großmaßstab eingesetzten Solarzellen mit Umwandlungsraten von knapp über zehn Prozent, einen fast zehnprozentigen Gewinn darstellt. Also werden Solarzellen bei weiterhin intensiver Forschung hin zu höherer Effizienz noch dominanter werden. Außerdem brauchen die thermischen Solarkraftwerke für hohe Leistung die direkte Sonnenstrahlung, also blauen Himmel, während die Fotovoltaikanlagen auch bei leichter Bewölkung, beispielsweise bei hohen Eiswolken, wie sie häufig auch über Wüsten vorkommen, fast dieselbe Leistung liefert.

\section{Bürgerenergie gegen große zentrale Energieinfrastrukturen}

Energiewende in der Wüste ist aus Industriesicht geschrieben und nimmt daher aus meiner Sicht zu wenig Bezug zur Basis, die für die ersten Erfolge der erneuerbaren Energien verantwortlich ist: die Umweltbewegung in den Demokratien der Industrieländer. Ohne das Engagement vieler

\footnotetext{
2 Interessant auch vor dem Hintergrund, dass etwa Saudi-Arabien über Jahrzehnte internationale Klimaschutzverhandlungen massiv behindert hat.
}

Bürgergenossenschaften wäre die extreme Kostendegression bei Fotovoltaik- und Windenergieanlagen an Land nicht möglich gewesen. So hat das im Jahr 2000 verabschiedete Erneuerbare-Energien-Gesetz $(E E G)$ in Deutschland wesentlich zu die-

ihre Wirtschaftlichkeit für den Golf-Kooperationsrat (Noch-Ölstaaten auf der arabischen Halbinsel) schwärmt geradezu von den hohen Potenzialen und den neuen Arbeitsplätzen, berichtet aber auch mit Sorge von der hohen Volatilität des Angebots. ${ }^{4}$

\section{Der Übergang zu einem zweiten solaren Zeitalter hat jetzt sogar in den Ölländern begonnen und ist aufgrund des Paris Agreement unumkehrbar geworden.}

sen geringeren Kosten beigetragen, weil für die Massenproduktion und damit die Verbilligung von Solarzellen dieser teilgeschützte Markt notwendig war (Eisenbeiß und Goetzberger 2011). Aber auch das Paris Agreement konnte erst dank der niedrigen Preise für Solar- und Windstrom geschlossen werden, weil die Regierungen nicht mehr befürchten mussten, dass ihre Wirtschaft den Abschied von den fossilen Brennstoffen nicht verkraften würde. ${ }^{3}$

Etwas drastisch formuliert könnte man schlussfolgernd sagen (und dazu hat mich das Buch angeregt, das ich sehr gerne las, weil es aus dem Inneren der Dii berichtet): Die grüne Bewegung hat das Geschäftsmodell der großen zentralen Energieinfrastruktur ins Wanken gebracht und in einigen westlichen Demokratien zum Teil schon beseitigt. Gleichzeitig hat aber durch das Scheitern der Mittelmeerunion die politische Unterstützung für Desertec nicht ausgereicht. Daher ist der zentrale Wunsch von Desertec, Wüstenstrom im großen Maßstab nach Europa zu liefern, zumindest zunächst gescheitert. Neue zentrale Energieinfrastrukturen, jetzt mit Sonnenenergie, wurden in den fast ausschließlich noch autoritär regierten Staaten im sonnenscheinreichen MENA fast ohne Bürgerbeteiligung, aber durch die Dii gestützt, auf den Weg gebracht. Die extreme Kostendegression für die Fotovoltaik wird auch den Traum mancher dieser Staaten, sich durch die Nutzung der Kernenergie leichter zu industrialisieren, als zu teuer entlarven, wie es van Son im Buch auch andeutet.

Eine Studie der International Renewable Energy Agency (IRENA 2016) über die Erneuerbaren-Energien-Potenziale und

\section{Die Solarzelle, Ursprung der Energiewende}

Das Buch Energiewende in der Wüste zeigt, dass der Übergang zu einem zweiten solaren Zeitalter jetzt sogar in den Ölländern - von Dii mit befördert - begonnen hat und aufgrund des Paris Agreement unumkehrbar geworden ist. Dafür spricht auch das viel geringere Konfliktpotenzial von erneuerbaren Energien, da viele Rohstoffkonflikte wegfallen: Weil die Sonne auf alle scheint und der Wind an allen Küsten bläst, hat jedes Land einen Großteil der Energierohstoffe selbst. Diese „Friedensdividende“ wird allerdings im Buch nicht thematisiert.

Es hat weit über 100 Jahre gedauert, bis der 1887 von Heinrich Hertz entdeckte Fotoeffekt und seine korrekte physikalische Interpretation durch Einstein im Jahr 1905 (wofür er 1921 den Nobelpreis erhielt) zur Solarzelle als Herzstück der zukünftigen Energieinfrastruktur für die meisten Regionen geführt haben.

\section{Literatur \\ Eisenbeiß, G., A. Goetzberger. 2011. Von klugen Köpfen und Katastrophen: die Entwicklung der Photovoltaik in Deutschland. GAIA 20/4: 236-242. DOI: 10.14512/gaia.20.4.6. \\ IRENA (International Renewable Energy Agency). 2016. Renewable energy market analysis: The GCC region. Abu Dhabi: IRENA. \\ Williams, J., G. Kromer, J. Weingart (Eds.). 1977. Climate and solar energy conversion. Proceedings of a IIASA workshop. Laxenburg: International Institute for Applied Systems Analysis. http://pure.iiasa.ac.at/734 (abgerufen 14.09.2020).}

3 Aus der Sicht des Klimatologen setzt globale Klimaschutz allerdings 30 Jahre zu spät ein.

4 Für mich überraschend wird in der Studie die Dii nicht erwähnt. 